\title{
Acerca del vocablo suicidio: preexistencia del acto humano de darse muerte respecto de la posibilidad de su nominación
}

\author{
Lorena Cecilia López Steinmetz ${ }^{1}$ \\ Universidad Nacional de Córdoba / CONICET, Argentina
}

INTRODUCCIÓN

Una afirmación general y que encuentra amplia anuencia entre los investigadores es que el vocablo suicidio fue de acuñación tardía. A partir de ese punto consensual, empiezan a encontrarse ciertas divergencias en cuanto al origen, a la etimología y a la datación de los primeros usos de vocablos referidos a prácticas suicidas. Esos puntos divergentes son los que se abordarán en este escrito. Cabe señalar que el adentramiento en el campo de interpretaciones ensayadas por diversos investigadores acerca de los motivos de la larga inexistencia lingüística en la nominación del acto de darse muerte, devela una exponencial multiplicación de discrepancias. No obstante lo interesante que puede resultar el análisis de ese último aspecto de la problemática filológica-si se quiere- del suicidio, la misma excede a los límites de este trabajo.

\footnotetext{
Para correspondencia, dirigirse a: Dra. Lorena Cecilia López Steinmetz (cecilialopezsteinmetz@unc.edu.ar), Instituto de Investigaciones Psicológicas, Universidad Nacional de Córdoba y Consejo Nacional de Investigaciones Científicas y Técnicas (IIPsiUNC-CONICET). Enfermera Gordillo esquina Enrique Barros, s/n, Ciudad Universitaria. CP: 5000. Córdoba, Argentina.
} 
Como se señaló, el mayor consenso investigativo indica que hasta antes del siglo XVII las menciones del vocablo suicida o de vocablos análogos fueron nulas o prácticamente nulas. Tal es así, que el citado vocablo habría hecho su aparición recién hacia mediados del siglo XVII (Barraclough \& Shepherd 1994; Clemente y González 1996; Corominas y Pascual 1986; Daube 1972; Grisé 1982; Van Hooff 1990; entre otros). Sin embargo, la fecha exacta del primer uso del término suicidio es, según Leenars (1995), aún una pregunta abierta.

Precediendo al siglo XVII, lo que los investigadores constataron con seguridad fue la ausencia de un vocablo único que designase al acto y al agente suicida. Esa ausencia constituye lo que se distingue en este trabajo como el obstáculo lingüístico en la designación del suicidio, el cual afectó tanto a las lenguas clásicas de Occidente como a las semíticas e indoeuropeas (Cohen Agrest 2012). En efecto, tal como lo estudió Daube (1972), la inexistencia de vocablos que denoten el suicidio se corroboró tanto en lenguas antiguas, tales como el hebreo, el griego y el latín, como en lenguas modernas, tales como el francés, el inglés y el alemán.

\section{DESARROLLO}

Hasta hace unos años, importantes referentes de este campo de estudio aceptaban que la primera mención registrada del término suicida fuese la que atribuyeran Barraclough \& Shepherd (1994) a un teólogo y moralista de mediados del siglo XVII, llamado J. L. Caramuel. Por ejemplo y al respecto, Hinojo Andrés (1998) coincidió con Van Hooff (1990) en sus primeros estudios, al explicar que Caramuel era el creador del neologismo suicidio.

Pero años después del estudio de Barraclough \& Shepherd, Murray (1998) divulgó el hallazgo de una mención anterior del vocablo suicida, la que habría sido plasmada en tiempos medievales. Sin embargo, a esa aseveración no le faltaron objeciones. Conociendo el contexto histórico de ese hallazgo, Cohen Agrest (2012) afirmó que al parecer no hay ninguna obra de la Edad Media donde conste el sustantivo suicidium para designar el acto como tal. Por ello, en opinión de la autora, lo que habría descubierto Murray serían, en realidad, rastros del empleo del término suicida, pero que designaban solo al agente del acto.

No obstante, Murray no reparó en connotaciones de ese tipo. En cambio, reivindicó su descubrimiento, cuya mención del vocablo suicida informó 
haberla hallado en un escrito monástico de finales del siglo XII, Contra quatuor labyrinthos Franciae, cuyo autor fue Walter de Saint Victor (como se citó en Murray 1998). Ese autor fue un teólogo rigorista y ultramontano, en cuya obra pretendió atacar y desprestigiar a famosos teólogos de su época y a los filósofos antiguos, entre ellos, a Séneca. Con el objetivo de denigrar a quienes decidían acabar con sus propias vidas, Walter de Saint Victor creó dicho vocablo con el mismo componente que homicida, fratricida, parricida, "como si se tratara de acciones no sólo equiparables sino peores" (Hinojo Andrés 2010, p. 61). Estas últimas connotaciones señaladas, y el valor con que se creó y utilizó el término, no escaparon al análisis de Murray (1998), quien afirmó al respecto que "en un punto, jugando con términos latinos, [Walter de Saint Victor] compara desfavorablemente el auto-asesinato con el fratricidio, una palabra latina establecida"2 (p. 38). Todo ello hizo que Murray no se limitase a señalar el hallazgo que constituía esa temprana mención, que venía a anteceder a las demás referencias halladas hasta entonces del vocablo suicida, sino que fue más allá, al ponderar el uso hecho allí del término, como índice del punto de colisión que por aquél entonces se jugaba entre los sistemas morales estoico y cristiano en torno del problema de la muerte voluntaria.

Por su parte, Edward Philips, en la edición del año 1662 de su diccionario The New World of English Words, reivindicaba para sí la invención de la palabra suicide (como se citó en Leenaars 1995). Sin embargo, el primer diccionario inglés en incluir el vocablo había sido Glossographia, de T. Blount, en el año 1656 (como se citó en Barraclough \& Shepherd 1994). Hecho que Philips no desconocía, no obstante lo cual, omitió su referencia. (Barraclough \& Shepherd 1994)

Pese a los diversos hallazgos mencionados, predominó otra referencia, que suele ser la más popularizada cuando se indica el primer uso del vocablo suicidio para designar al acto como tal. Dicha referencia es la que Al Álvarez proclamó haber descubierto años antes en un texto de Sir Thomas Browne, titulado Religio Medici, escrito en 1635 y publicado en 1642 (como se citó en Leenaars 2010). Desde entonces, comúnmente se relaciona a Browne con la creación del término.

Sin embargo, ocurrió que durante los años que mediaron entre la escritura y la publicación de la obra de Browne, el neologismo suicidio se difundió en lengua inglesa a través de una versión publicada en 1637 del Satyricon

\footnotetext{
2 En el original: "At one point, punning with Latin terms, he compares the self-killer unfavourably with the fratricida, an established Latin word". (Murray 1998, p. 38)
} 
de Petronio (como se citó en Cohen Agrest 2012). Luego, el uso del término se extendió paulatinamente, siendo asentada oficialmente su entrada en el Oxford English Dictionary en 1651 (como se citó en Leenaars 1995). Desde el inglés se difundió a las lenguas continentales, aunque se demoró un tanto su aceptación definitiva. En francés fue utilizado por primera vez por el Abad Desfontaines en 1737 (como se citó en Hinojo Andrés 2010). En 1762, la Academia Francesa de la Lengua incluyó también el suicidio dentro de su vocabulario, al hacerlo figurar en el Dictionaire de l'Academie (como se citó en Clemente y González 1996). Mientras que en castellano, la primera inclusión del vocablo suicidio en el Diccionario de la Real Academia Española data de 1817, si bien denotando por entonces solo el acto, mientras que en la edición de 1822, el mismo vocablo figura como sustantivo y como adjetivo (como se citó en Corominas y Pascual 1986). Aunque además se tienen noticias de que en España este término habría hecho su aparición por primera vez en la crítica a Voltaire que realizó fray Fernando de Ceballos en 1772, dentro de su obra La falsa filosofía y el ateísmo (Martí 1982, como se citó en López García, Hinojal Fonseca y Bobes García 1993). También tardó en difundirse en Italia y no se incorporó a los diccionarios hasta muy avanzado el siglo XIX. (Hinojo Andrés 2010)

Ahora bien, Cohen Agrest (2012) señaló que en 1632 Browne habría acuñado el neologismo suicidio, en su forma compuesta formada a partir del latín sui (de sí mismo) y caedes (asesinato). Al respecto, otros autores como Giner Jiménez (2010) informaron que el neologismo suicide "procede de las palabras latinas sui y occidere" (p. 29). O también, por otra parte se halla la referencia de la Real Academia Española (2001), que afirma respecto del término suicidio, que es este una voz formada a semejanza de homicidio, proveniente del latín: sui, de sí mismo, y caeděre, matar. Sea como fuere, a Cohen Agrest (2012) le interesó señalar que Browne habría concebido la citada acuñación compuesta del neologismo suicidio a los fines de distinguir el self-killing cristiano, conceptuado como totalmente condenable, del suicidium pagano pero noble de Catón. Según informó la autora, este término compuesto, pseudoclásico, se habría empleado en inglés a fines de sortear la connotación negativa de términos en uso por ese entonces -también en inglés- en los lenguajes europeos, tales como "selfmurder" y "self-slaughter" (p. 72). Ello es coincidente con lo expuesto por otros investigadores, como Leenaars (1995), quien, además de los términos citados, señaló que por aquél entonces también se usaban los términos "self-destruction" y "self-killing" (p. 347) para designar el acto de darse muerte. En similar sentido, Barraclough \& Shepherd (1994), al examinar el origen y usos del vocablo suicidio, hallaron que dicho término fue usado inicialmente por quienes cuestionando las prohibiciones y dogmas de la 
Iglesia defendían, en cambio, el derecho individual a darse muerte. Con fundamento en lo anterior, concluyeron que el suicide inglés, que apareció recién hacia mediados del siglo XVII, había surgido como parte de los debates sobre la moralidad-inmoralidad del acto suicida. Al respecto, el término suicide resultaba neutral, y a diferencia de los vocablos que le antecedieron (self-murder, self-homicide) evitaba el estigma resultante de equiparar dicho acto con un crimen.

En relación con lo anterior y continuando en la profundización de la controversial historia de la génesis del vocablo en cuestión, se hallan aseveraciones de que la moderna palabra suicidio y sus variantes fonéticas, común a muchas lenguas modernas de Europa, en realidad no habría derivado de un compuesto latino (Cohen Agrest 2012), ni de un pretendido término latino tal como suicidium, ya que este nunca habría existido en el latín clásico, en el latín tardío, ni en el latín medieval (Hinojo Andrés 2010; Morin 2008). Aún más, ese término nunca habría podido existir porque "los hablantes de la lengua de Roma nunca formaron los compuestos con un primer elemento pronominal, ni con suus-sui, ni con ningún otro" (Hinojo Andrés 2010, p. 60). Coincidentemente, Cohen Agrest (2012) señaló que "el prefijo suus no se usaba en expresiones compuestas" del latín clásico (p. 71). En similar orientación, Van Hooff (1990) explicó que, dado el carácter opuesto a las normas gramaticales latinas de construcción de palabras que ostenta la locución suicida, su aparición solo podría ser postclásica en función de unir pronombre y verbo. En este sentido, es que en latín y a oídos romanos, el vocablo significaría literalmente: dar muerte a un cerdo (Cohen Agrest 2012), o matador de cerdos, pero no matador de sí (Daube 1972; Van Hooff 1990). Dicho de modo similar, para un conocedor de la lengua Lacio, suicidum/suicida solo podría aludir a la matanza del cerdo, el matarife. (Hinojo Andrés 2010)

Las anteriores aserciones son opuestas a la tendencia más generalizada, que entre tantos otros expuso también Minois (trad. 2001), y que consistió en afirmar que el término suicidio procede del latín sui que significa sí mismo y cidium que significa matar; para quien, en consecuencia, suicidio significaría matarse a sí mismo. Esta tendencia generalizada de remontar el origen del vocablo suicidio a un compuesto latín, con términos que remiten a sí mismo y a matar, también es la que sustenta actualmente la Real Academia Española (2001), tal como se citara párrafos más arriba.

Lo que hoy conocemos como suicidio, fue objeto de encuadramientos retóricos muy variados en distintas culturas, fuera como crimen, pecado, sacrificio, enfermedad, etc. Asimismo, en el plano filológico, a lo largo de los siglos se aludió a la muerte voluntaria recurriendo a numerosas y variadas expresiones compuestas y/o a circunlocuciones. No obstante el 
reconocimiento de esa diversidad retórica y lingüística, Morin (2008) afirmó que lo que se encuentra a modo de constante es una subyacente tendencia general al silenciamiento del suicidio, lo cual coincide con aseveraciones de diversos autores, tales como Cohen Agrest (2012) y Pérez Jiménez (2011), entre otros. Al respecto, de acuerdo con Pérez Jiménez (2011), el verdadero tabú en el que se debe reparar residiría en la "férrea ley del silencio que la sociedad se impuso a sí misma" (p. 14), a modo de pacto colectivo para mirar hacia otro lado, investido de respeto o de desprecio, pero que "en definitiva esquiva la mirada del suicida y de sus familiares como cómplices indirectos o sospechosos de estar afectados por el mismo mal”. (p. 14)

El rastreo de la evolución histórica de las expresiones con las que se aludió a la muerte voluntaria que hizo Cohen Agrest (2012), dejó entrever un pasaje progresivo que fue desde descripciones descarnadamente concretas que se apegaban a la literalidad de los hechos, hacia expresiones un poco más abstractas y eufemísticas. Ello, toda vez que no se hubiese optado en su lugar por el silenciamiento. Pero aun habiendo descripto dicha evolución, la autora reconoció que ambos tipos de expresiones coexisten paralelamente en armonía hasta hoy, especialmente en el lenguaje coloquial, aunque no así en el discurso científico. Tanto el recurso a la literalidad descriptiva como a las expresiones eufemísticas pueden entenderse como correlatos necesarios del problema de la inexistencia de un vocablo único y unívoco que designase al acto y al agente que se da muerte a sí mismo. Como parte integrante del mismo contexto problemático, puede ubicarse la práctica de utilizar términos emparentados desde cierta lógica pero que, al proceder de determinados contextos históricos y culturales, arrastran también indisociablemente otros sentidos que exceden "al de una aséptica noción de muerte autoinfligida" (Morin 2008, p. 159). Parte de estos recursos, también problemáticos, serán ejemplificados a continuación presentando algunas de las expresiones utilizadas en diversos momentos y contextos histórico-culturales.

En la Antigua Grecia, desde el siglo V a. C. ya se servían de vocablos que completaban su sentido con el agregado de una especificación, de expresiones compuestas o locuciones perifrásticas tales como morir por la propia mano. Un ejemplo de esta última expresión se encuentra en Historia Ecclesiastica, de Eusebio, donde fue empleada para describir el suicidio de Pilatos (como se citó en Morin 2008, p. 162). Como señala Cohen Agrest (2012), lo interesante de esta expresión es que con ella se presentó al suicidio por vez primera como una forma de morir y no como una forma de matar. Llamativamente, el surgimiento de esta expresión no fue resultado de una novedosa reflexión filosófica sobre el tema, sino que, en esencia, resultó “del simple hecho de que el empleo del veneno fue perfeccionado hasta tal grado 
de refinamiento, que una porción, en su justa dosis y a mano, aseguraba un fin relativamente pacífico" (pp. 69-70).

También en Roma se utilizaron expresiones tales como morir por mano propia para aludir al suicidio. Al respecto, el aporte de Grisé (1982) consistió en destacar que el uso clásico de la expresión latina sua manu para referir al suicidio remitía a la connotación de potestad que se le asignaba a manus. Con ello, el suicidio se presentaba como el acto lícito y deliberado de un hombre libre y, por tanto, consciente de sus actos. En este marco, solo respecto de quienes no eran dueños de sí mismos (esclavos, soldados, acusados) se podía discutir la licitud del suicidio. Justamente, fue al romano Cicerón a quien se le atribuyó la acuñación de la más antigua frase nominal con que se aludió al suicidio: mors voluntaria. Acuñación trascendente, que se inserta en este punto de los desarrollos porque reclamaba para esta forma de morir el reconocimiento de su naturaleza deliberada. En similar sentido, entre las expresiones que cubrieron la ausencia del término requerido para nominar el acto de darse muerte, también se puede citar otra que era empleada en la antigua Roma. La expresión mortem sibi consciscere, era empleada por los juristas romanos y enfatizaba también el carácter decisional del acto y, por lo tanto, el rol de la voluntad y la conciencia del suicida. (Grisé 1982)

Las expresiones referenciadas posibilitaron que la concepción del suicidio, como una forma de matar, se fuera refinando hasta llegar a indicar una forma de morir. El pasaje de esta práctica por el tamiz de la subjetivación del hecho aportó que "se trata de una especificación del acto que da cuenta de una subjetividad operando en un estadio más reflexivo". (Cohen Agrest 2012, p. 71)

Sin embargo, antes de llegar a esa refinación terminológica, también se emplearon otros vocablos que se distinguieron por su carácter indefinido. Según Timothy Hill (como se citó en Van Hooff 2007), en la élite romana, ello estaría explicado por el hecho de que el suicidio no era distinguido como una especie distinta de muerte. Ejemplo del empleo de términos de carácter indefinido fue un término griego, cuya forma latina es authéntes, que podía remitir en su significado tanto a un "self-killer", como a alguien que hubiese asesinado a un miembro de su propio linaje (Van Hooff 2007). Otros autores señalaron que este vocablo griego tuvo la particularidad de haber remitido, inicialmente, al crimen producido al interior del grupo de parentesco de pertenencia, aunque su uso más generalizado habría sido en tanto término relacional. Esto es, en el sentido de indicar una vinculación, que podía ser eventualmente parental, entre sujetos que tenían muertos en común, unos en carácter de víctimas y otros de victimarios. (Belfiore 2000 como se citó en Morin 2008) 
Otra expresión griega utilizada para hacer referencia al suicidio se incorporó a mediados del siglo II de la era cristiana, con un vocablo asociado al suicidio salvaje, que en su forma latina es biothanatos o biaiothanatos. En ésta, su forma latina, el vocablo oculta su etimología y pareciera plantear un binomio vida/muerte, pero en realidad a lo que remite es a una forma violenta de morir, que en los inicios de su uso excedió a la muerte por propia mano (Daube 1972; Murray 1998; Van Hooff 1990). Por tanto, biothanatos, que inicialmente era traducible como morir con violencia, englobaba a cualquier tipo de muerte brutal. Pero también en este caso, fue el componente de libre elección de la muerte propia el que hizo que este vocablo que resultó de gran importancia en los siglos XVI y XVII, prontamente se erigiera en un término técnico que acotó la amplitud de su significado inicial a algo así como morir por violencia autoinfligida. Las controversias que suscitó este término entre los escritores del Renacimiento hizo que rápidamente llegara a designar al suicidio en general. (Cohen Agrest 2012)

Las referencias a personas que se dan muerte a sí mismas pueblan muchísimos textos medievales, pero la enunciación de tales actos quedaba encuadrada, finalmente, bien bajo la perífrasis sui homicida, bien con referencia al término desperatus. En relación con este último, el término desperatio o desesperatio, no constituyó precisamente un término técnico debido a que fue portador de muchos sentidos. Entre ellos, su rastreo por numerosos textos da cuenta de la connotación que comportaba una amplia gama de sentidos bélicos. Otra acepción del término es la que se puede denominar sentido médico, conforme a la cual, desperatio remitía al cuadro del desahucio (Morin 2008). No obstante, el sentido más referenciado es el teológico, el cual condenaba el hecho de que el objeto de la desesperanza fuese la merced divina. Esta referencia a la desperatio, según Schmitt (1976 como se citó en Morin 2008), representa el maître-mot del suicidio medieval. Además, al provenir del ámbito teológico, reconduce el homicidio de sí mismo a un pecado gravísimo, el que se constituía cuando se desesperaba de la merced divina, ya que en última instancia ello implicaba una negación de los poderes de Dios, así como de la vida eterna. Schmitt entendió que la desesperatio no era un sentimiento, ni un estado físico, sino un vicio, la duda de la misericordia divina, la convicción de no poder ser salvado. El suicidio representaba así la victoria del diablo, que desasía de esperanza al alma. Asimismo, Ros Montalbán (1997) informó que durante los siglos VIII a IX, en los países anglosajones, donde se castigaban la mayoría de los suicidios, la desesperanza no solo no era consideraba una justificación, sino que en términos morales y debido a las connotaciones religiosas citadas, era vista como la forma de suicidio más grave. En esos tiempos fue cuando la Iglesia católica emprendió la difusión de la confesión individual, con lo cual 
esta institución religiosa se manifestaba como el paso intermedio necesario para la comunicación con Dios. En ese contexto, el suicidio por desesperatio presuponía por parte del sujeto que lo cometía, una imposibilidad de ser perdonado por sus pecados. Esto es lo que le ocurrió a Judas, quien por una parte, pecó contra Dios al dudar de su bondad, y por otra, pecó contra la Iglesia al dudar de su poder de intercesión. (Giner Jiménez 2010)

Como se señaló, el sentido teológico no agota en absoluto los usos y acepciones del término desperatio. No obstante, dado que muy a menudo el término fue utilizado directamente, sin especificar si la referencia remitía o no al origen teológico, la resultante fue que prácticamente se estableció una sinonimia entre desesperados y suicidas. De modo que, "en un punto, el lenguaje teológico de la desperatio parece teñir todas las referencias medievales a los suicidas". (Morin 2008, p. 160)

Por último, se hará mención a la evocación que hizo Murray (2000) del término latino suspendiosi, con el que se designó a los suicidas durante largo tiempo. Este vocablo hacía referencia, en primera instancia, a quienes morían ahorcados, pero pronto designó a quienes se ahorcaban a sí mismos. De acuerdo a Murray, este vocablo expandió su campo semántico hasta ser usado para designar a las personas suicidas independientemente del método de autolisis que hubieran utilizado.

\section{CONCLUSIONES}

La revisión etimológica plasmada en este escrito, junto al breve análisis de las variadas prácticas -reflejadas en diversos encuadramientos retóricoscon que a lo largo de la historia se dio tratamiento a lo que hoy se conoce como suicidio, apoyan el entendimiento de que el suicidio fue concebido primero como una forma particular de matar. Es decir, como una forma de asesinato, cuando no también como una forma de crimen, asociado a la violencia. De allí que un capítulo aparte de análisis lo constituiría el abordaje de lo que se puede denominar como las legislaciones sobre el suicidio. Solo postreramente el suicidio fue concebido como una forma de morir, o como un caso particular de violencia ejercida contra uno mismo. Esto es, como un acto de muerte voluntaria en el que convergen sujeto y objeto de la acción violenta. 


\section{REFERENCIAS BIBLIOGRÁFICAS}

Barraclough, Brian y Daphne Shepherd. 1994. A necessary neologism: The origin and uses of suicide. Suicide and Life-Threatening Behavior 2: 113-126.

Clemente, Miguel y Andrés GonzÁlez. 1996. Suicidio: una alternativa social. Madrid: Biblioteca Nueva.

Cohen Agrest, Diana. 2012. Por mano propia. Estudio sobre las prácticas suicidas. Buenos Aires: Fondo de Cultura Económica.

Corominas, Joan y José Antonio Pascual. 1986. Diccionario crítico etimológico castellano e hispánico. Madrid: Gredos.

Daube, David. 1972. The linguistics of suicide. Philosophy and Public Affairs 4: 387-437.

Giner JimÉnez, Lucas. 2010. Diferencias en la conducta suicida. Estudio comparativo entre los intentos de suicidio y suicidio consumado. Tesis Doctoral, Universidad Autónoma de Madrid, Facultad de Medicina, Departamento de Psiquiatría. Madrid, España.

Grisé, Yolande. 1982. Le suicide dans la Rome Antique. París: Les Belles Lettres.

Hinojo Andrés, Gregorio. 1998. "Suicidio": barbarismo y perversión. Jueces para la Democracia: Información y Debate 32: 12-13.

2010. Las designaciones de la muerte voluntaria en Roma. Hápax 3: 57-72.

LeenaArs, Antoon A. 1995. Suicide. En Hannelore Wass y Robert A. Neimeyer (eds.). Dying: Facing the facts. $3^{\mathrm{a}}$ ed., pp. 347-384. Washington D.C.: Taylor \& Francis.

2010. Edwin S. Shneidman on suicide. Suicidology Online 1: 5-18 [en línea]. Disponible en http://www.suicidology-online.com/articles2010.html

López García, María Beatriz, Rafael Hinojal Fonseca y Julio Bobes García. 1993. El suicidio: aspectos conceptuales, doctrinales, epidemiológicos y jurídicos. Revista de Derecho Penal y Criminología 3: 309-411.

Minois, Georges. Trad. 2001. History of suicide: Voluntary death in western culture. Lydia G. Cochrane, trad. Baltimore: The Johns Hopkins University Press.

Morin, Alejandro. 2008. Sin palabras. Notas sobre la inexistencia del término 'suicida' en el latín clásico y medieval. Circle de Clásicos y Modernos 12: 159-166.

Murray, Alexander. 1998. Suicide in the Middle Ages: The violent against themselves. Vol. 1. New York: Oxford University Press.

2000. Suicide in the Middle Ages: The curse on self-murder (Vol. 2). New York: Oxford University Press.

Pérez Jiménez, Juan CARlos. 2011. La mirada del suicida. El enigma y el estigma. Madrid: Plaza y Valdés.

Real Academia Española. 2001. Diccionario de la lengua española (DRAE). $22^{\circ}$ ed. [en línea]. Madrid: Autor. Disponible en http://www.rae.es

Ros Montalbán, Salvador. 1997. La conducta suicida. Madrid: ELA.

Van Hooff, Anton. 1990. From autothanasia to suicide. Self-killing in Classical Antiquity. New York: Routledge.

2007. From voluntary death to self-murder: The dialogue on self-killing between Antiquity and Christian Europe. En John Hilton y Anne Gosling (eds.). Alma parens originalis? The receptions of classical literature and thought in Africa, Europe, The United States and Cuba, pp. 269-290. Germany: Peter Lang AG, International Academic Publishers. 